\title{
A Bilateral Electro-Hydraulic Actuator System to Measure Dynamic Ankle Joint Stiffness during Upright Human Stance
}

\author{
S. M. Forster, R. Wagner, and R. E. Kearney \\ Department of Biomedical Engineering, McGill University, Montreal, Canada
}

\begin{abstract}
A bilateral electro-hydraulic actuator system has been developed to measure the dynamic joint stiffness of the human ankle during standing. The apparatus consists of two foot pedals that are each connected to an electro hydraulic rotary actuator. Transducers are incorporated to measure the position and torque of each actuator, the angle of the ankle with respect to the foot plate, and the positions of the knee and hip. Nonlinear identification techniques will be used to determine the reflex contribution to the overall torque.
\end{abstract}

Keywords - posture, reflex, intrinsic, stretch, ankle, torque

\section{INTRODUCTION}

Human standing is a complicated task in which the nervous system must coordinate sensory input to generate an appropriate musculoskeletal response. Even during quiet stance, the central nervous system continuously receives sensory information from the visual, vestibular, and somatosensory systems and processes this information to maintain a stable upright posture.

External perturbations destabilize the body, which the nervous system must then counteract. In the anterior posterior direction, two strategies have been described [1]. Large perturbations evoke the "hip strategy" involving large trunk rotations and smaller ankle rotations. In contrast, smaller external perturbations evoke an "ankle strategy" during which the body acts as an inverted pendulum, rotates about the ankle joint, and is controlled by the ankle muscles.

Dynamic joint stiffness is the relationship between the position of a joint and the torque acting on that joint. It is important in postural control since it is what resists the external perturbations before the voluntary muscle contractions intervene. Dynamic stiffness has two components: 1) intrinsic stiffness due to passive joint properties as well as the mechanical properties of the active muscle, and 2) reflex stiffness due to the changing muscle activation mediated by the stretch reflex.

The role of the stretch reflex during upright stance is still unclear. Collins and Deluca [2] analyzed the center of pressure during quiet stance and found evidence for both open-loop and closed-loop control of posture. Winter et al. [3], on the other hand, found no evidence of feedback control. They concluded that postural control cannot be reactive since the velocities and accelerations of the body during quiet stance are all below the sensory thresholds, and thus, it must be passive [4]. It has also been suggested that a feedforward control system may be in use $[5,6]$.

Several methods are presently used to separate intrinsic and reflex components of muscle torque. These include electrical stimulation, anesthesia, and system identification techniques. Our laboratory has made extensive use of a non-linear, parallel cascade identification scheme [7] to determine the relative contributions of intrinsic and reflex mechanisms in normal subject and neurological patients. However, these experiments have all been conducted with subjects lying prone with no requirement to maintain postural stability.

This paper describes a new experimental apparatus that has been developed to allow us to identify intrinsic and reflex contributions to ankle torque in freely standing subjects. We first describe the design requirements, then describe the apparatus, and conclude with some preliminary results describing its performance.

\section{DESIGN REQUIREMENTS}

The design problem was to create a device which would enable us to study human ankle dynamics during standing. In addition to the study of basic intrinsic and reflex mechanism, the apparatus must be designed to enable the study of these mechanisms in response to different functional requirements, such as with and without vision, with and without external support, with changes in the base of support, and with loads applied to the subject.

This led to the following design requirements:

1. The system must be capable of imposing random perturbations to ankle position over a range of motion of $\pm 6^{\circ}\left(0^{\circ}\right.$ being when the foot pedal is horizontal).

2. The system must have a frequency response that is greater than the range of frequencies present during postural sway. A bandwidth of $20 \mathrm{~Hz}$ was felt to be more than adequate. 
3. The maximum torque required for each actuator was calculated to be $350 \mathrm{Nm}$ and a maximum speed of $20 \mathrm{rad} / \mathrm{s}$

4. The apparatus must be able to measure the torque and position of each actuator during perturbations.

5. The position of the foot with respect to the actuator must also be measured to account for the possibility of heel lift. The knee and hip angles need to be measured in order to determine whether the body is in fact acting as an inverted pendulum. The position of the feet and ankles during standing must also be considered. The distance between the feet must be adjustable within the range of $15 \mathrm{~cm}-$ $40 \mathrm{~cm}$.

6. The rotation of the actuator shaft must be in line with the axis of rotation of the ankle joint. Therefore the distance of the foot pedal from the axis of rotation must also be adjustable

7. The safety of the subject must be ensured at all times.

\section{CONSTRUCTION}

\section{A. Actuator Construction}

Fig. 1 shows a schematic diagram of the completed apparatus. It consists of two electro hydraulic actuators coupled directly to foot pedals. The rotation shaft of the actuator is in line with the axis of rotation of the ankle.

The rotary actuators (Rotac 26R-2 $1 \mathrm{~V}$ ) are controlled by proportional servo valves (Moog D681-4718). The actuators operate at a maximum pressure of $20690 \mathrm{kPa}$ and can generate a maximum torque of $583.1 \mathrm{Nm}$. The angular position of each foot pedal is measured using a conductive plastic $5 \mathrm{k}$ rotary potentiometer (Maurey Instruments 112P19). The potentiometer has a linearity of $\pm 0.5 \%$ over a theoretical electrical travel of $340^{\circ} \pm 5^{\circ}$. The signal from the potentiometer is conditioned using custom built electronic modules which allow the gain and offset to be adjusted. These modules are calibrated to have a sensitivity of 10 $\mathrm{V} / \mathrm{rad}$. A positive angle is measured during dorsiflexion whereas a negative angle is measured during plantarflexion.

Torques are measured using two flanged reaction torque sensors (Lebow 2110-5k). These transducers have a capacity of $565 \mathrm{Nm}$ and a torsional stiffness of 103941 $\mathrm{Nm} / \mathrm{rad}$. These signals are conditioned using custom built electronic modules which allow the offset to be set as desired. These modules are calibrated to a sensitivity of

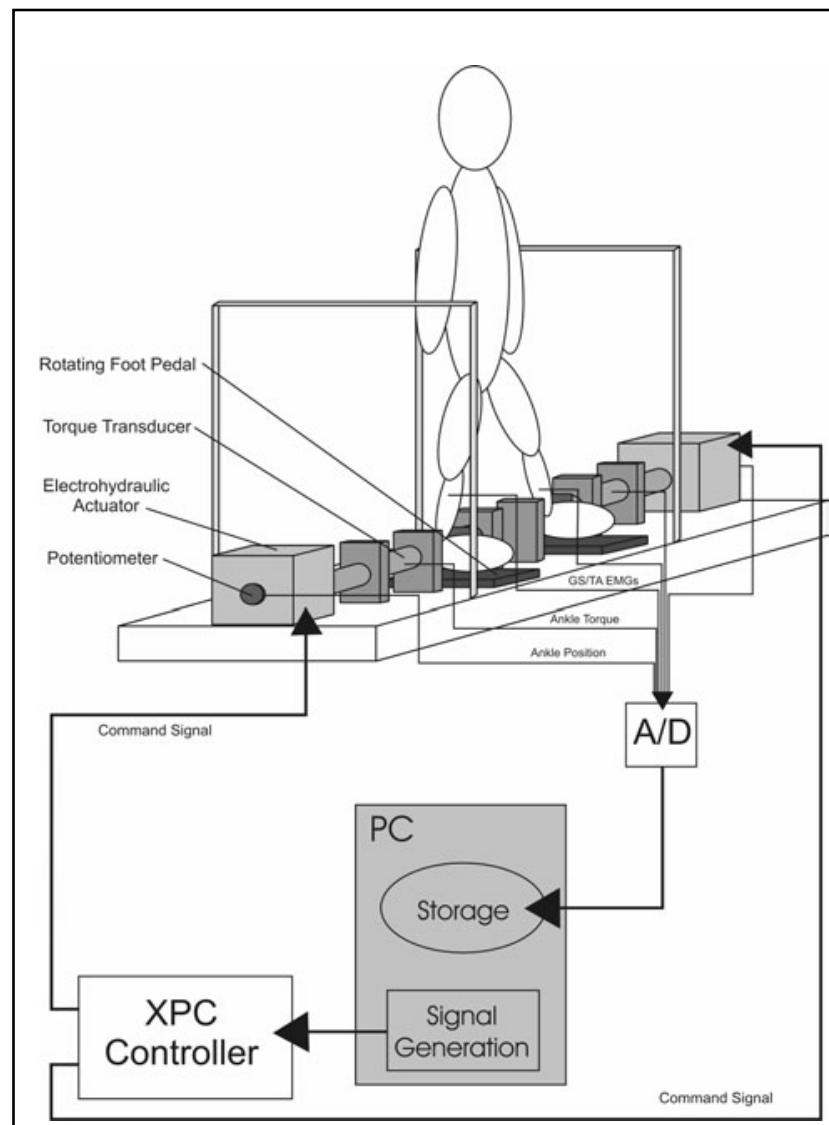

Fig. 1. Schematic of the bilateral actuator

$0.05 \mathrm{~V} / \mathrm{Nm}$. A positive torque is measured during dorsiflexion whereas a negative torque is measured during plantarflexion.

\section{B. Safety}

To prevent injury to the subject, five safety mechanisms were incorporated:

1) The actuator shafts both have a cam which allows a rotation of about $\pm 20^{\circ}$, when the rotation exceeds this range, the cam mechanically activates a directional valve which blocks the hydraulic pressure going to the servo-valve;

2) Two other steel cams rotate with the actuator shafts and block the rotation when the cam comes in contact with bolts used as mechanical stops, which are set by the individual conducting the experiment;

3) The servo-valves are equipped with fail-safe mechanisms so that if the external power supply is interrupted, the spool moves to its centered position therefore stopping the rotation of the actuator; 
4) Handrails to the left and right of the subject are in place for the subject to grab on to if ever they feel unstable;

5) A full body harness (Jelco 740) is worn by the subjects and attached to the steel crossbars in the ceiling.

\section{Transducers}

The right foot pedal is equipped with an ultrasonic sensor (Baumer Electric UNDK 30U6113/S14) to measure heel lift. It is mounted under the foot pedal and a small hole has been machined in the foot pedal to allow the sound pulse to bounce off the subject's heel.

On the right hip and knee, there will be two angular sensors (MicroStrain Inc. FAS-G-M) to measure the hip and knee angles. These will be attached to the subject's skin using medical grade, two-sided tape.

\section{Controller}

The servo-valve controller was developed using SIMULINK (The MathWorks, Inc.) and compiled on the host computer using the Real-Time Workshop Toolbox for MATLAB. It used a MATLAB-based XPC real-time digital signal processing system. The target computer is equipped with a 16 bit, 8 channel A/D card (Computer Boards PCIMDAS1602/16) and a 16 bit, 6 channel D/A card (Computer Boards PCIM-DDA06/16). The host loads the DSP code to the target using Ethernet. For these experiments, a controller was created which uses proportional position control for each actuator.

\section{PERFORMANCE EVALUATION}

\section{A. Methods}

The dynamic performance of the new actuator was assessed using the two types of position input that will be used initially to examine joint stiffness:

1) Pulse Perturbations in which the command input was a rapid pulse with a width of $40 \mathrm{~ms}$ and an amplitude of 0.015 rads. This permits the reflex and intrinsic components to be dissociated on the basis of latency since, due to the reflex delay, the intrinsic response will be completed before the reflex response begins.

2) Random Perturbations: where the command input is a random signals having a bandwidth $0-20 \mathrm{~Hz}$ and peak to peak amplitude of 0.015 rads.

The experimental code was developed using MATLAB (The MathWorks, Inc.). The computer workstation communicates with the external analog to digital (A/D) and

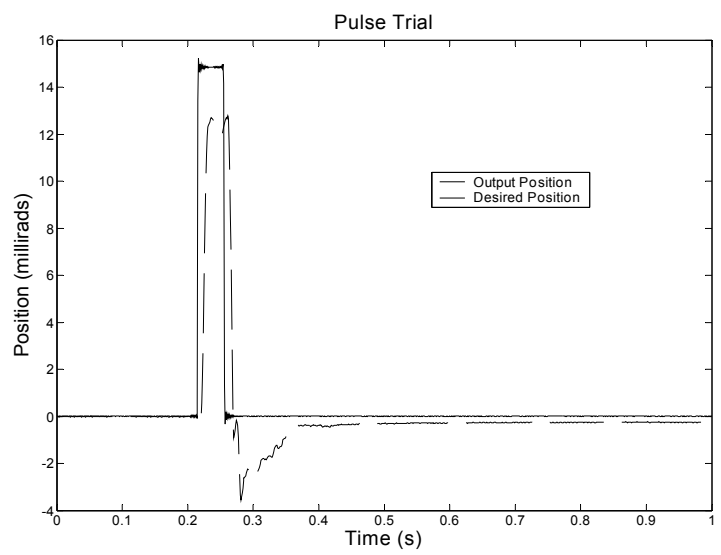

Fig. 2. Position command input and the resulting output for a $40 \mathrm{~ms}$ pulse stimulus

digital to analog (D/A) devices via a general purpose interface bus (National Instruments 488.2 v1.60). All data signals were sampled at a frequency of $1 \mathrm{kHz}$ using a dynamic signal acquisition card (National Instruments 4472). The signals were then decimated to $50 \mathrm{~Hz}$.

Data presented here were acquired with a normal human subject standing on the device. A $60 \mathrm{~s}$ pulse trial and a $60 \mathrm{~s}$ random trial were acquired.

\section{B. Pulse Experiments}

Fig. 2 shows a pulse input and the resulting position of the subject's left ankle. Pulse rise time was $<15 \mathrm{~ms}$ and the total pulse length was $50 \mathrm{~ms}$. This should be adequate for reflex identification. There is a small undershoot at the end of the pulse whose origin we are still investigating.

\section{Random Experiments}

Fig. 3 shows the command input and the corresponding
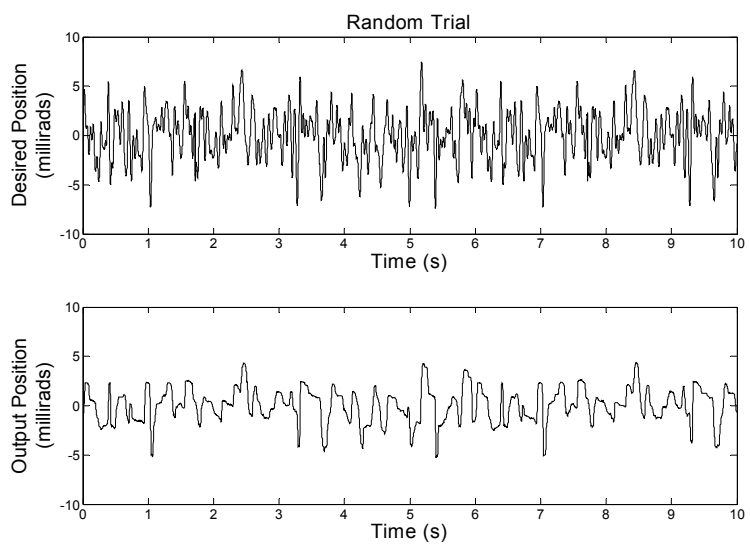

Fig. 3. Desired position input and the resulting output for a random stimulus 

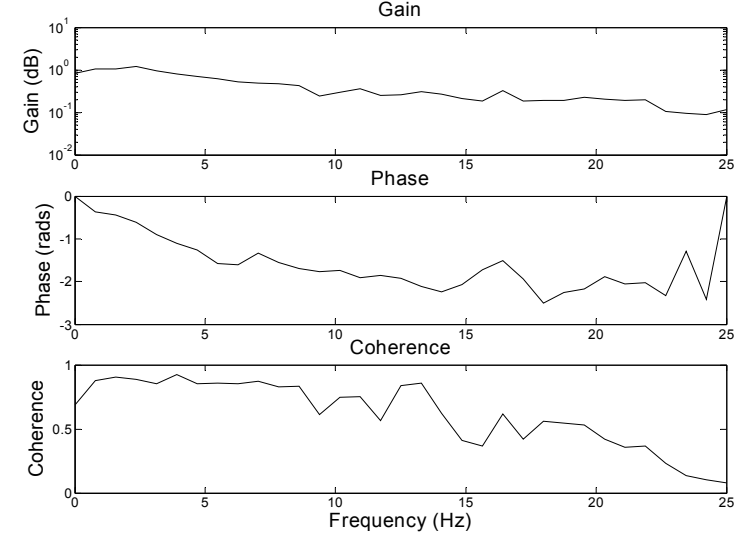

Fig. 4. Frequency response of the position output

left ankle position for a random trial. It is evident that the low-pass dynamics of the actuator have eliminated some of the high frequency content of the command input.

Fig. 4 shows the frequency response of the actuator computed from the random data of Figure 3. The coherence squared is greater than 0.5 up until $20 \mathrm{~Hz}$ indicating that the response is well correlated with the input to that frequency. The gain is flat at low frequency and then begins to roll off at $3 \mathrm{~Hz}$ and is down by $20 \mathrm{~dB}$ by about $20 \mathrm{~Hz}$. The phase lag increases linearly until $20 \mathrm{~Hz}$ where it begins to oscillate. These results indicate that that the useful bandwidth of the device extends from $\mathrm{DC}$ to $20 \mathrm{~Hz}$.

\section{DISCUSSION}

This experimental apparatus will be used measure dynamic ankle joint stiffness, and the relative contributions of reflex and intrinsic mechanisms, in humans during upright stance.

Subjects will be trained to stand quietly while undergoing small amplitude stochastic perturbations. Each ankle will be driven by independent perturbations to minimize net effect on the body lean.

Trials will be run (i) with/without vision; (ii) with/without additional support in the form of a handle in front of the subject; (iii) with the ankle at different positions to reduce the base of support; (iii) with different loads applied to the back of the subject in increase the effective gain of the stretch reflex needed for stability.

Intrinsic and reflex stiffness in standing subjects. Since the analysis focuses on ankle dynamics, it does not depend upon the body acting as an upright pendulum. However, movements at the knee could cause problems by changing the length of the gastrocnemius, which attaches above the knee. We do not anticipate significant movement at the knee but will monitor knee angle in any case. Should significant knee movements occur, we would use a custom-fitted orthosis to lock the knee in an extended position. We note that this experimental paradigm will also require "closedloop" identification methods since the responses evoked by perturbations to ankle positions can cause changes in ankle position. [8]

Experiments will be conducted using random and pulse perturbations to subjects under three trial conditions: 1) One foot pedal remains stationary and horizontal while the other moves according to the command signal; 2) Both foot pedals move according to the command signal; 3) One foot pedal moves according to the command signal and the other foot pedal moves according to the negative of the command signal.

\section{ACKNOWLEDGMENTS}

Special thanks to Hank Leung, Ayman Afanah, Fadi Akache, Pascal Fallavollita, Ziad Nabhani, Becky Shaw, Alison Duncan, Fred Arseneault, Alex Whitehouse, and Mackenzie Baker. Supported by a grant from the Canadian Institutes of Health Research (CIHR)

\section{REFERENCES}

[1] Nashner, L.M., McCollum, G. "The organization of human postural movements: a formal basis and experimental synthesis," The Behavioral and Brain Sciences, Vol. 8, pp135-172, 1985.

[2] Collins, J.J., De Luca, C.J. "Open-loop and closed-loop control of posture: A random-walk analysis of center-of-pressure trajectories," Experimental Brain Research, Vol. 95, pp308-318, 1993.

[3] Winter, D.A., Patla, A.E., Prince, F., Ishac, M., Gielo-Perczak, K. "Stiffness control of balance in quiet standing," Journal of Neurophysiology, Vol. 80, pp1211-1221, 1998.

[4] Winter, D.A., Patla, A. E., Ishac, M., Gage, W. "Motor mechanisms of balance during quiet standing," Journal of Electromyography and Kinesiology, Vol. 13, pp49-56, 2003.

[5] Gatev, P., Thomas, S., Kepple, T., Hallett, M. "Feedforward ankle strategy of balance during quiet stance in adults," Journal of Physiology, Vol 514(3), pp915-928, 1999.

[6] Fitzpatrick, R., Burke, D, Gandeva, S.C. "Loop gain of reflexes controlling human standing measured with the use of postural and vestibular disturbances," Journal of Neurophysiology, Vol. 76(6), pp3994-4008, 1996.

[7] Kearney, R.E., Stein, R.B., Parameswaran, L. "Identification of intrinsic and reflex contributions to human ankle stiffness dynamics," IEEE Transactions on Biomedical Engineering, Vol. 44(6), pp493-504, 1997.

[8] Tung, J.Y., Kearney, R.E. "Closed-loop estimation of intrinsic and reflex contributions to ankle stiffness," Proceedings of the $2^{\text {nd }}$ Joint EMBS/BMES Conference, October 2002, Houston, TX, pp.2550-2551. 